\title{
Hilbert's Hotel as Purely Possible and as an Actuality
}

\section{Gilead A* \\ Department of Philosophy, University of Haifa, Israel}

*Corresponding author: Amihud Gilead, Department of Philosophy, University of Haifa, Israel, Email: agilead@research.haifa.ac.il

\section{Research Article}

Volume 3 Special Issue 1

Received Date: November 13, 2020

Published Date: December 10, 2020

DOI: $10.23880 /$ phij-16000S1-005

\section{Abstract}

It was George Gamow who publicized the world of the fascinating thought-experiment that he called "Hilbert's Hotel," whose author was the eminent mathematician David Hilbert and which Gamow interestingly associated with the Big Bang cosmology and the expansion of the universe. The number of the rooms in Hilbert's Hotel is infinite and they are always fully occupied. Nevertheless, this fantastic hotel can receive any number of new guests at any time and can find a vacant room for each of them. According to Gamow and some interpreters, Hilbert's Hotel demonstrates how an actual infinite is possible and that it is not an absurd idea. In contrast, others have interpreted Hilbert's thought-experiment as meant to show the contrary, namely, that an actual infinity is impossible and simply an absurd idea. In this paper, I explain how Hilbert's Hotel can be purely possible and actual as well.

Keyword: Actual infinite(ies); Mathematical possibilities; Mathematical objects or entities; Possibilism; individual pure possibilities; Panenmentalism; the Big Bang; New inflation cosmology; David Hilbert; George Gamow; Frederick Hoyle; Baruch Spinoza

\section{Introduction: The Thought-Experiment Known as Hilbert's Hotel}

The fascinating thought-experiment, known as Hilbert's Hotel and whose author was the eminent mathematician, David Hilbert, goes as follows: There is a grand hotel comprising an infinite number of rooms. Even though this Grand Hotel is always fully occupied, it is universally known as never refusing any new guest who asks for a room at any time. Suppose that a new visitor arrives at the hotel at a time in which it is fully occupied (which is the usual case) and asks for a vacant room. There is no problem in complying with such a request. As the hotel has an infinite number of rooms, the manager would ask the guest in room no. 1 to move to room no. 2 , and the guest in room no. 2 to move to room no. 3 , and so on and so forth, simply to admit the new guest to room no. 1. There is no vacant room in this hotel, and yet it is always possible to receive any new guest as well as many other guests. Suppose now that an infinite number of new guests would like to book rooms in the Hotel. The proprietor has no problem in granting their wish. He has simply to move the guest in room no. 1 to room no. 2, the guest in room no. 2 to room no. 4 , that in no. 3 to no. 6 , and so on to infinity. In this way, as, following Cantor, "the infinity of even numbers is exactly as large as the infinity of all numbers" (Gamow 1953 [1947], p. 28), all odd-numbered rooms can become vacant and the infinite number of new guests can be easily accommodated in them. Hence, new guests ad infinitum would have rooms enough in this miraculous hotel, which is always fully occupied. If our universe is like a Hilbert's Hotel, then new galaxies, for instance, even infinite in number, have "rooms" enough in it.

This thought-experiment was strongly associated with George Gamow, the father of the Big Bang cosmology, who named this experiment "Hilbert's Hotel." ${ }^{1}$ The aim of this

1 Hilbert 2013, p. 695 and 730. See Kragh 2014. As for Gamow's version, see Gamow 1953 (1947), pp. 25-34; and Gamow 1952, pp. 28-29 (included in Chapter II, entitled "The Great Expansion," in the section entitled "the 


\section{Philosophy International Journal}

thought-experiment was to demonstrate that "in the world of infinity a part may be equal to the whole" (Gamow 1953 [1947], p. 28) and that the notion of an actual infinite is possible and does not lead to absurdities, however counterintuitive it may be ${ }^{2}$. Notably, Cantor preceded him in showing that an actual infinite is possibly and actually exists ${ }^{3}$. Gamow clearly associated it with his cosmological theory (Gamow 1952, pp. 21-29).

Cantor distinguished between three kinds of actual infinities: The actual infinite arises in three contexts: first when it is realized in the most complete form, in a fully independent other-worldly being, in Deo, where I call it the Absolute Infinite or simply Absolute; second when it occurs in the contingent, created world; third when the mind grasps it in abstracto as a mathematical magnitude, number, or other type. I wish to make a sharp contrast between the Absolute and what I call the Transfinite, that is, the actual infinities of the last two sorts .... (Cantor, Gesammelte Abhandlungen, p. 378; translated in Rucker 1982, p. 9)

As I will explain below, the actual infinite as actual or "when it occurs in the created world," needs to be contingent, and the transfinite numbers are also mind-independent as the mind discover them and does not invent them. Nevertheless, as I will explain below, like other numbers, transfinite numbers, as objects of pure mathematics, are not actual entities but mathematical pure possibilities, which are also mind-independent. Only their representations in our mind are mind-dependent. However, pure mathematical objects as such are not abstractions, they are rather particular, individual pure possibilities, which are mind-independent.

theory of the expanding universe"). Gamow's approach to the problem of the actual infinite is clearly incompatible with Hilbert's view about the possibility of an actual infinite (Hilbert 1998 [1926], p. 201: "Our principal result is that the infinite is nowhere to be found in reality"). Hilbert considered such an infinite set as a conceivable or possible universe but not as an actual) one (Ellis, Kirchner, and Stoeger 2004, p. 927). Stoeger, Ellis, and Kirchner (2008) state as following: "From the point of view of cosmology itself, it would be very helpful if we could trust in the conclusion that an actualized mathematical infinity is physically impossible. ... If, instead, there emerges a clear indication that actual infinite sets are possible, that would be mathematically disappointing." Cf. Kragh 2009, p. 541.

2 For a recent treatment of the problem of an actual infinity, see Puryear 2014.

3 "It was Georg Cantor who, in the late 1800s, finally created a theory of the actual infinite which by its apparent consistency demolished the Aristotelian and scholastic 'proofs' that no such theory could be found. Although Cantor was a thoroughgoing scholar who later wrote some very interesting philosophical defenses of the actual infinite, his point of entry was a mathematical problem" (Rucker 1982, p. 7). This begins a most intriguing story: Rucker draws an analogy between the type of construction Cantor was working with and the Koch curve, which is discussed in length in Benoit Mandelbrot's Fractals, in which he explained "why there is reason to think of the Koch curve in its infinite spikiness as being a better model of a coastline than any of its finitely spiky approximations" (op. cit., p. 9).
My approach to the problem of Hilbert's Hotel rests upon a special possibilist realism about individual pure possibilities, which has been called "panenmentalism." ${ }^{4}$ For reasons that will be detailed below, this realism challenges the notion of possible worlds (hence, the pure possibilities under discussion are individual) and, especially, any kind of actualism.

\section{Individual Pure Possibilities, Actualism, and Possible Worlds}

What are individual pure possibilities and for what purposes they are necessary?

Individual pure possibilities are primary or fundamental entities whose existence does not depend upon any spatiotemporal and causal condition or restriction and upon anything actual. These entities determine or fix the identities of all individual actual entities, that is, actualities. The existence of actualities, in contrast, depends upon spatiotemporal and causal conditions or restrictions.

Primary, fundamental, or basic entities are those that are absolutely irreducible to other entities and without which nothing could exist. Anything that exists is possible, otherwise it could not have existed. Anything that exists is thus an existing possibility, actual or purely possible. Given that my main interest in the modal term "possibility" or "possibilities" is ontological and that I defend a realist approach to individual pure possibilities, I refer to possibilities as substantive modal entities or objects (to be distinguished from modal properties) rather than to modal operators or quantifiers. Given that, what makes an existent possible from the outset is the individual pure possibility of that existent. What makes an existent possible from the outset is what distinguishes it, ontologically and epistemologically, from any other entity, purely possible or actually possible.

The term "pure" is similar to the one in the term "pure mathematics" to be distinguished from "applied mathematics". Purely mathematical entities are purely mathematical individual possibilities, whereas the entities of applied mathematics are actual entities-actualities-of these possibilities.

4 Panenmentalism is my original metaphysics, which I suggested and elaborated on since 1999. It treats some of the basic problems of philosophy, to begin with the psychophysical problem, and spreads over the major fields of philosophy, especially ontology, epistemology, psychological philosophy, philosophy of science, theory of literature and fiction, and ethics. In each of these fields, panenmentalism mainly studies individual pure possibilities and their relations (in general, relationality). For the panenmentalist publications and the applications of panenmentalism to some major issues in the philosophy of science, consult Gilead 1999, 2003, 2009, 2010, 2011, 2013a-b, 2014a-d, 2015a-e, 2016a-e, and 2020. 


\section{Philosophy International Journal}

The individual pure possibilities discussed in this paper are mainly mind-independent and hence they are quite different from the "pure possibilities" that some phenomenologists, to begin with Edmond Husserl, have assumed and which are clearly mind-dependent or ideal entities. "Pure", in my terms, indicates an exemption from any spatiotemporal and causal property, condition, or restriction, whereas "actual" implies yielding to such properties, conditions, or restrictions. Furthermore, "pure" means exemption from, and ontological and epistemological independence of, anything actual.

Even though individual pure possibilities are nonactual, this does not mean that they are not actualizable. Furthermore, an individual pure possibility is a non-actual or unactualized possibility also to the extent that something must be left of any individual pure possibility that remains pure and not actualized despite its actualization as an actuality. The reason for this is that any actuality could have changed or been different and yet remains one and the same entity, and, thus, no actuality can exhaust all that pertains to its individual pure possibility. No actuality can completely, entirely actualize its individual pure possibility, which, thus, necessarily remains pure, at least to some extent. Any actuality is simply the spatiotemporally restricted part of its individual pure possibility which is, thus, always more comprehensive or "larger" than its actuality. Hence, the distinction (which is not a separation) between individual pure possibilities and their actualities, whenever such exist in fact, is always maintained.

Note that no two individual pure possibilities can be identical. So-called or apparent "identical" pure possibilities are one and the same possibility. As absolutely exempt from any spatiotemporal and causal property, two individual pure possibilities that apparently are "identical" cannot exist in the same time at different places or at the same place in different times, and because of this, there would be nothing to make any difference or distinction between them. This would render them indiscernible from each other. As indiscernible, they must be identical as one and the same individual pure possibility. In other words, the time-honored Leibnizian principle of the identity of indiscernibles must hold true for individual pure possibilities.

As each actuality has an individual pure possibility of its own, which it does not share with any other entity, this pure possibility fixes or determines the identity of that actuality. Under whatsoever actual circumstances, yielding to spatiotemporal and causal conditions or restrictions, and under any possible change, actualities maintain their identities owing to their individual pure possibilities from which they are inseparable.
The reason for this inseparability or necessary connectivity is that without the individual pure possibility, or separately from it, any actuality could not be possible, could not exist from the outset. Secondly, any actuality is in fact the spatiotemporally and causally restricted or conditioned part of its individual pure possibility. Each pure possibility thus comprises its actuality as such a conditioned and restricted part of it, provided that such an actuality exists. If the actuality does not exist, the individual pure possibility in consideration exists independently of the fact that there is no actuality of it. In any event, no actuality is separable from its individual pure possibility, from what fixes the identity of the actuality in question.

Pure possibilities are, first and foremost, substantial individual entities or their parts. Other individual pure possibilities are states and tropes of such entities, whereas properties, being general or universal, pertain to the ways in which individual pure possibilities relate to one anotherto their relationality. We should not mistake relationality for relatedness. As we shall see, relationality rests upon the difference between the relating entities (i.e., individual pure possibilities), whereas relatedness rests upon the similarity or likeness of the related entities.

What are individual pure possibilities not? These possibilities are not abstract entities, haecceities, potentialities (and the like), Platonic Ideas, or possible worlds.

They are not abstract entities because, as I understand this term, such entities are abstracted out from actualities, whereas individual pure possibilities are entirely independent of actual reality and thus cannot be abstracted out from it. Abstraction requires criteria, according to which the abstraction is made and which are not abstracted out from actual reality, unless an infinite regress were brought about. Dependence on such criteria, on the one hand, and on actualities, on the other, renders abstract entities as unsuitable to serve as individual pure possibilities, which are fundamental entities. Furthermore, actual entities are concrete existents, whereas individual pure possibilities are specific or particular entities, which, obviously, are not general. In contrast, abstract objects are generalized from actual, concrete entities.

Individual pure possibilities are not haecceities. As each such possibility comprises all that is possibly open to an entity under one and the same identity, it is not a sort of an essence, let alone an actual essence; thus, an individual pure possibility is not a haecceity.

Individual pure possibilities are not potentialities, propensities, or dispositions. Individual pure possibilities are 


\section{Philosophy International Journal}

absolutely independent of actualities, whereas potentialities, propensities, and dispositions depend on actualities from which they drive their possibility and identity. For instance, the seed is a potential plant, and the seed gets its nature and identity from the actual plant. The same holds true for the disposition and propensity of the seed to be a plant. Hence, existing independently of and prior to the physical-actual, pure possibilities are not potentialities, propensities, or dispositions.

Individual pure possibilities are not Platonic Ideas either because these Ideas are universals, in which their tokens "take part" or which individual things "imitate" (and as Aristotle criticized these terms, they must be simply metaphorical), whereas individual pure possibilities are individual entities. Platonic Ideas are not possibilities. Instead, they are "paradigms" and "causes" of any other entities, namely, of their "tokens" or "namesakes". These "tokes" or "namesakes" are separate from the Ideas (this separation is the famous chōrismos because of which Aristotle harshly criticized Plato and the Platonists), whereas actualities are never separate from their individual pure possibilities.

Finally, individual pure possibilities are not possible worlds. Even though the possible-worlds conception is quite prevalent and accepted by possibilists as well as actualists, ${ }^{5}$ it is questionable, in any of its forms, for various reasons. It is not clear enough, and there are many controversies about it with no universal or long-standing consent. The problem of the epistemic accessibility from one world to another, especially from the actual world in which we live to any possible world, does not appear to have a satisfactory solution. Moreover, if possible worlds are possible ways, maximal or otherwise, of actual reality (the ways in which actual reality could have been), they must depend on it, and thus they cannot be appropriate to pure possibilities, which are entirely independent of actual reality. For this reason, many actualists have accepted the ideas of possible worlds and of possibilia at possible worlds, whereas I suggest a

5 Yet some recent actualists have dispensed with it, for instance, Jacobs 2010, Vetter 2011, and Fine 2012. Consider also Lowe 2006. Christopher Menzel defines actualism and possibilism as follows: "Actualists ... deny that there are any non-actual individuals. Actualism is the philosophical position that everything there is-everything that can in any sense be said to be-exists, or is actual. Put another way, actualism denies that there is any kind of being beyond actual existence; to be is to exist, and to exist is to be actual. Actualism therefore stands in stark contrast to possibilism, which . . . takes the things there are to include possible but non-actual objects" (Menzel 2014). Contrary to actualism, my possibilist approach distinguishes between two kinds of existents-that of individual pure possibilities and that of actualities. In my view, individual pure possibilities exist independently of actualities. Even though many actualists adopt the idea of possible worlds, all of them explicitly reject the existence of merely, purely possible individuals or particulars, which are entirely independent of the actual. possibilist alternative, completely realist about individual pure possibility and the ways in which they relate to each other, namely, their relationality, and, thus, entirely independent of any possible-worlds conception. As for merely possible worlds, entirely independent of anything actual, they, too, raise more problems than reasonable solutions, especially regarding the epistemic access to them. If, according to other views, possible worlds are propositions about actual facts or states that could have been different, again, they depend on actual facts, which render them inappropriate for my possibilist view. Hence, if we can dispense with the possible-worlds conception and find an adequate, clearer and simpler, alternative to it, we should take this possibility into consideration.

Indeed, individual pure possibilities are not susceptible to such problems and difficulties. They are clearer in comparison to the concept of possible worlds. To refer to these possibilities is quite simple. Strip any individual actuality of its spatiotemporal and causal conditions, and you are referring to and considering its individual pure possibility without being involved in any real difficulty or obscurity. To do so, all you need is to use your intellect and imagination. You can simply consider the identity of such an actuality in comparison with anything else you can imagine, regardless and independently of anything spatiotemporal or causal.

"Stripping" an individual entity of its spatiotemporal and causal conditions, restrictions, or properties is not abstracting, for no generalization is involved in such a "stripping" process, whereas abstracting requires generalization. A "stripping" process, in contrast, clearly uncovers and preserves the individual identity of the entity under consideration. The same holds true for comparing, regardless of anything spatiotemporal or causal, the entity under consideration with any other entity in order to consider its individual identity. Nothing of generalizing is involved in such a process, which is thus free from any abstracting. By no means does the outcome of this process depend upon the actuality that is relevant to the starting of the process, rather the contrary-the actuality under discussion depends on the outcome, which is an individual pure possibility. We start the process with that actuality in order to uncover what makes it possible from the outset, namely, to reveal the possibility on which it depends, ontologically and epistemologically, from the outset.

Any actuality is possible but not all possibilities are actual. Hence, there are many more individual pure possibilities than actualities and the whole realm of individual pure possibilities is larger and more comprehensive than its spatiotemporally and causally restricted part, which is actual reality as a whole. 


\section{Philosophy International Journal}

To sum up, individual pure possibility is what distinguishes, ontologically and epistemologically, an individual entity from any other entity, possible or actual, regardless of any spatiotemporal and causal conditions and independently of anything actual or empirical.

For what are individual pure possibilities good? In other words, are individual pure possibilities necessary and for what purpose? Individual pure possibilities are necessary for ontological and epistemological reasons. For ontological reasons, because without individual pure possibilities no actual existent would be possible-if any actuality were not purely possible from the outset, namely, prior to the spatiotemporal and causal conditions of its existence, it could have not existed at all from the outset. For epistemological reasons, because without individual pure possibilities we cannot identify, know, and understand many natural phenomena nor discover something new. When crystallographers observe or synthesize for the first time some unfamiliar even "absurd" crystals (quasicrystals, for instance)-whose constructions are incompatible with all that the crystallographers theoretically or empirically have known-they must have an access to the pure, theoretical possibilities of such strange, totally unfamiliar crystals. Such pure possibilities are purely mathematical. Think, furthermore, about nuclear physicists or chemists who observe or produce for the first time some strange transuranium atoms. These atoms are so heavy that they are expected, empirically and theoretically, to collapse or to be disintegrated within some milliseconds. Nevertheless, they sustain for quite a lot time and demonstrate some new chemical properties indicating a new element. These physicists must have some access to the individual pure possibilities of such atoms. Such possibilities can be discovered only by theoretical-mathematical means and not by any empirical, actual observations or experiments. Scientists would have not discovered the actual Higgs boson some years ago, if Higgs and two other theoretical physicists had not succeeded in their theoretical discovery of the pure possibility of such a boson (for which they gained the Wolf Prize). And there are many other examples.

As for our epistemic accessibility to individual pure possibilities, we always can use our free imagination to consider individual pure possibilities that are different from any entity with which we are familiar, of which we have been reported, or which have been described to us. Creative persons are especially able to do so. Original artists do so all the time. And the same holds true for inventors and discoverers. Ordinary people frequently use their imagination for thinking about such possibilities or to consider them while initiating projects, making plans, thinking about new solutions to some problems, considering the unexpected, thinking of something that is considered as improbable yet possible, and so on. Whenever we think or believe that things could be otherwise or different, we are, in fact, thinking about or considering individual pure possibilities and their general relations. However finite and limited our knowledge of individual pure possibilities and their general relations, this knowledge is always open for further expansions and progress.

In what follows, I will take a possibilist way, realist about individual pure possibilities, as this way is less traveled despite its surprising merits and novelties, whereas the actualist way, especially since the works of Quine, Goodman, and many others, has been much more traveled and has become, in many of its variations, much more popular and pertaining to the main stream.

There is a deeper reason for my preferring the possibilist option to the actualist one mentioned above. We, human beings, are not confined to actual reality as a whole and to actualities. Individual pure possibilities-as the objects of our planning, choosing, creating, predicting, and discovering - are vital for our life. Our imagination and intellect are certainly not confined to actual reality and they do not necessarily need to abstract possibilities out from it.

We need individual pure possibilities, real possibilities, to know and understand the world, ourselves, and our relationships with other people. No law can be said to hold without assuming possibilities and excluding others. Both the determinist and the fatalist believe that things could not be different or otherwise, and to believe so one must first assume possibilities to exclude some of them. All the more so are those who believe that things could be different and even entirely different; they must assume the existence of real possibilities even if such possibilities have never become actual. Every existent, every entity, is possible. There are no impossible entities. All the other modal categoriesexistence, contingency, and necessity-are all kinds of possibility.

Do non-actual, non-physical possibilities exist independently of our mind? There are, certainly, such possibilities, for instance, the entities or objects of pure mathematics are, in fact, mathematical individual pure possibilities. It is quite reasonable to consider purely mathematical entities as mathematical individual pure possibilities. In pure mathematics (to be distinguished from applied mathematics, as application and actualization are considered as synonyms), whatever is proved to be possible is, in fact, proved to be a purely mathematical existent, and if the proof is sound, this existent is also necessary. These purely mathematical entities are thus mathematical individual pure possibilities, whose existence is entirely independent of actual, physical reality and of any spatiotemporal and causal 


\section{Philosophy International Journal}

conditions. The existence of these discovered entities is mind-independent.

Individual pure possibilities are non-actual (even though they are actualizable); they are thus not subject to any spatiotemporal and causal conditions. In contrast, all physical entities, all actual possibilities, are subject to the same spatiotemporal and causal conditions or restrictions. Thus any actual thing, any actuality, is a physical entity, and any physical entity is an actual individual possibility-an actuality-different from any other actuality. Any actuality is an actualization of an individual pure possibility, which is distinguished or discernible from any other individual pure possibility. For this reason, any individual pure possibility serves as the identity of an actuality.

Physical reality is vitally dependentupon its mathematical individual pure possibilities and their universal relationality. Pure mathematics, as its name indicates, is independent of any applicability to anything actual-physical, whereas anything actual-physical is vitally dependent upon the applications of pure mathematics. Any physical entity, any actuality, is quantitative and must have quantitative and other arithmetical and geometrical properties. These properties are actualizations of the general or universal relationality of mathematical individual pure possibilities. Without the applications of pure mathematics, quantities and other arithmetical and geometrical properties make no sense. But the reverse is excluded, as pure mathematics is entirely independent of actual properties and of being applied to the actual-physical reality. Even if actual quantities and, hence, the actual-physical reality as a whole did not exist, pure mathematics, as comprising mathematical pure possibilities, would have existed as it is. Pure geometrical or arithmetical entities have all the meaning, sense, and significance they need without relying upon any actual quantities. The reverse, however, is simply impossible.

Furthermore, actual-physical entities are also dependent upon physical individual pure possibilities, for these possibilities determine and fix the identities of these actual entities. For instance, any actual particle depends for its identity upon its individual pure possibility, otherwise it would not have been possible from the outset. Physical individual pure possibilities are discovered by means of pure, theoretical physics, which our intellect and imagination construct. Thus, the pure possibilities of atoms and of subatomic particles (such as the Higgs boson, the omegaminus, and the like) were discovered, and necessarily so, independently and, in many cases, before the discovery of the actual particles. The theoretical discovery is of an individual pure possibility that determines and fixes the identity of the actual particle. Actual physical reality is thus dependent upon the pure possibilities that a purely physical theory discovers.
Whenever such a discovery is correct and complete, physical-actual reality is as this theory truly describes it, based upon the purely possible grounds of this reality. There are individual pure possibilities that theoretical physics or chemistry discovered in the past and yet these possibilities (for instance, aether, caloric, and phlogiston) happened to remain unactualized and the theories that discovered them proved to be empirically wrong. The contrary is the case of physical individual pure possibilities that have been actualized and empirically confirmed as actualities. In each of these examples, the relevant individual pure possibilities exist independently of the actual-physical reality and of empirical observations. Nevertheless, only experiments and empirical observations can tell whether we conceive the possibilities that happen to be actualized and whether the relevant physical theory is empirically valid.

\section{Hilbert's Hotel as Purely Possible and as Actual}

All the reasons with which the former Section supports realism about individual pure possibilities are valid for the individual pure possibility of Hilbert's Hotel and for the actual Hilbert's Hotel as well. Let me emphasize now some of the most relevant of them. Yet before doing that, I would like to draw the attention of the reader to the difference between individual pure possibilities that comprise and are open to infinite number of possibilities, on the one hand, and individual pure possibilities that comprise and are open to a finite or limited number of possibilities, on the other. Hilbert's Hotel pertains to the first kind of individual pure possibilities. In other respects, all I have said above about individual pure possibilities and actualities is strictly valid for the individual pure possibility and the actuality of Hilbert's Hotel as well.

As no actual entity, no actuality, exhausts all the possibilities that are open to it, such is obviously the case of Hilbert's Hotel: all the new arrivals, possible guests, however infinite their number, can be accommodated at one and the same Hilbert's Hotel. There are rooms in it for all actual guests as well as for all possible ones. In any case, actual or possible, both the numerical identity and the qualitative one of this Hotel are not changed. At any moment, this very Hotel is fully booked and yet, at the same time, it is open to all new, possible guests. The same holds true for the modes of the accommodation of this Hotel. At any moment, the Hotel comprises an infinite number of rooms and its capability of accommodating an infinite number of guests is not exhausted.

Nevertheless, the size (Cantor's "cardinality") of the infinite set of the purely possible rooms of this Hotel is larger than the size of the infinite set of its actual rooms at a particular time. Such is the case, because each of the actual 


\section{Philosophy International Journal}

rooms is possible too, but there are further purely possible rooms, which are non-actual at that particular time. In any event, Hilbert's Hotel as an individual pure possibility is more comprehensive or larger than Hilbert's Hotel as an actuality. This Hotel as an actuality is a conditioned or restricted part of Hilbert's Hotel as purely possible. As actual-physical, this part is spatiotemporally and causally restricted, whereas the most comprehensive Hilbert's hotel, the Hotel as an individual pure possibility, is not restricted by any spatiotemporal and causal condition or limitation. The actual infinite part of the Hotel is comprised in its purely possible whole. What remains of it as non-actual is always open for possible actualizations, in rooms or in guests, in different times and under different circumstances. No actual occupied rooms, no actual booking, and no actual number of guests can exhaust the possibilities, in rooms and in guests, that must be always kept open for this fantastic Hotel.

\section{Saving the Possibilities of Hilbert's Hotel and of Actual Infinity as Well}

In 1972, challenging a paper by Pamela Huby (1971) about the Hilbert's Hotel thought-experiment, N. W. Boyce argues as follows (my comments are added within square brackets):

Mrs. Huby... claims that if we assume that the World is infinite, we involve ourselves in many paradoxes. She cites, as one of these paradoxes, "Hilbert's Hotel" ... If we assume, Mrs. Huby claims, that the World is infinite, we must accept "Hilbert's Hotel" as a real possibility [i.e. an actual possibility; an actuality]. But however counterintuitive "Hilbert's Hotel" may seem, it describes a logically possible state of affairs [i.e. a purely possible state of affairs; note that the logically possible is one kind amongst many kinds of pure possibilities]. However, "Hilbert's Hotel," is more than a merely logically possible World [i.e. a purely possible universe]. In fact "Hilbert's Hotel" describes, metaphorically, the structure of the Universe as it is conceived [i.e. as it is conceived as actual] by the "Steady State" Cosmology-that is if the "Steady State" theory of the Universe is true, then, we are living in something very like "Hilbert's Hotel." ... Thus "Hilbert's Hotel" is no mere mathematical fiction, but, may be the world we actually live in [i.e. the actual world in which we live]. (Boyce 1972, p. 68)

Nowadays the steady state cosmology is no longer accepted, and a new modified version of the inflation cosmology has gained more support. Should this cosmological theory be established as true, this means, as I will explain, that Hilbert's Hotel is the actual universe in which we live. Furthermore, Boyce's defense of this fascinating thought-experiment should be supported by the panenmentalist reasons that I have introduced above. Hence, what is printed in bold type within the square brackets in the cited passage above is my "translation" of Boyce's terms into panenmentalist ones. It appears that Boyce limited mere, pure, or non-actual possibilities to the logical or purely mathematical ones, while there are many other kinds of pure possibilities in additions to the logical and mathematical ones.

As an actuality is a restricted part of its pure possibility, any actual infinite is a spatiotemporally and causally restricted part of its pure possibility. As Hilbert's Hotel is such an actuality, there are always free, unoccupied rooms that can be occupied-can be actualized so-at any time. The relevant pure possibilities are such empty rooms and new guests to accommodate them in these rooms. Hilbert's Hotel as an individual pure possibility is an infinite set whose subset is an actual infinite set. This demonstrates nicely how panenmentalism is perfectly compatible with the idea of actual infinity in general and with that of Hilbert's Hotel in particular.

Hilbert's Hotel may leave many of us, as Pamela Huby was, feeling discontent. There appears to be something paradoxical about it: after all, if the Hotel, despite its infinite number of rooms, is fully occupied, how on earth can each new, would-be guest be given a vacant room? If the Hotel is fully occupied in fact and there is no actual vacant room, how on earth can there be an infinite number of vacant rooms for an infinite number of new guests? And what about the infinite space in which an infinite number of new-born galaxies have always had new rooms? We should reconcile two facts that seem to be entirely incompatible-all of the Hotel's rooms are in fact occupied and yet there is always a vacant room available for any new guest.

The panenmentalist approach distinguishes between two kinds of Hilbert's Hotel: a purely possible Hilbert's Hotel and an actual-physical one, which in turn is the limited and conditioned actuality of the purely possible one. As purely possible, this Hotel is larger, bigger, and more comprehensive than the actual Hotel; yet each of them has respectively a purely possible or actual infinite number of rooms, all of the actual ones are permanently occupied and yet each of them can accommodate new "guests" - galaxies and the likeinstead of the actual ones. Except for the purely possible and actual infinity, which characterize the case of Hilbert's Hotel, similar is the part-whole relationship, which is valid for any individual pure possibility and its actuality-the actuality is the restricted and spatiotemporally and causally conditioned part of the individual pure possibility as a whole. The conditioned actual part exists within the whole individual pure possibility and is included in this possibility (hence, the immanent nature of pure possibilities). Note 


\section{Philosophy International Journal}

that no actuality can exhaust its pure possibility; there is more to the pure possibility of a thing than to its actuality, as an actual entity. Any actuality could have been different in respect of spatiotemporal and causal circumstances or conditions with no change in its identity. Despite all the possible changes of the actuality, its identity, numerically and qualitatively, remains one and the same, as this identity is simply an individual pure possibility, which is unchangeable. For instance, the laptop on which I am writing this paper could have been very far from here, under quite different circumstances and taking part in quite a different causal chain, and yet it would be one and the same laptop.

The realm of pure possibilities is maximally infinite; it includes anything purely possible. A strict, conditioned part of this realm is the physical-actual, which is spatiotemporally and causally conditioned or restricted. Hence, the physicalactual is a conditioned or restricted part of the purely possible; and the purely possible is "wider" or "more comprehensive" than the physical-actual, which is included in the purely possible.

The infinite space of Hilbert's Hotel is not only actual, it is not only the infinite actual expanding universe in which all of us possibly exist; this hotel is also purely possible. As actual, it takes a spatially and causally conditioned part of the purely possible universe. The actual space (all the actual rooms in the Hotel) is, like anything actual, limited, confined, and restricted, whereas the space, rather the realm, in which any pure possibility of any galaxy is included and any actuality of this galaxy as an actuality has a spatiotemporal and causal location, is maximally infinitely wide. Yet, the actual universe is infinite, though smaller than the purely possible universe, whose infinity is larger or more comprehensive. This should not surprise us, as, according to the Hilbert's Hotel thoughtexperiment, there is room enough for bigger and smaller infinities (Gamow 1953 [1947], p. 33).

Given all these panenmentalist considerations, it appears that there is nothing really paradoxical or absurd about Hilbert's Hotel. May I remind the reader that the theory of quantum mechanics was considered as counter-intuitive, paradoxical, and even absurd, and yet nowadays nobody would seriously reject it on such grounds, all the more since this theory has been undoubtedly empirically confirmed and its predictions are entirely accurate. At present, such appears to be the state of Hilbert's Hotel-however counter-intuitive and paradoxical this thought-experiment may appear, it rests on solid grounds, all the more so since the cosmology related to it may be established as empirically true. Indeed, a purely possible infinite is valid and sound, provided that some extraordinary pure possibilities are not excluded on the grounds of dogmas and restrictive ways of thinking. However paradoxical and counter-intuitive a phenomenon- actual or purely possible-may be, this alone is not a sound reason to reject the phenomenon in question.

As open-minded, panenmentalism makes an attempt to liberate our way of thinking from dogmatic confinements, among which there are commonsensical ones, and from any restriction to actual reality and to our random empirical experience as well. Panenmentalism attempts at saving as many pure possibilities as possible. Hence, it attempts to save the pure possibilities of actual infinities. I believe that one of the reasons why Huby and many others who have excluded the possibility of an actual infinite, is that they have excluded the happy idea that the part may be equal to the whole (namely, each of them can be infinite), which Gamow happily endorsed. Panenmentalism, too, endorses this pure possibility and finds it quite enlightening.

Note that on quite different, actualist grounds, Baruch Spinoza endorsed the idea of an actual infinite (Letter 12 of 20 April 1663, Spinoza 1985, pp. 200-205; an actual infinite is mentioned on pp. 204 and 205). He thought that only because of one's confinement to random experience and one's mistaking imagination, one could not conceive the idea of actual infinity. One's wrong conception of numbers, for instance, is a bad reason for excluding actual infinity (see Gilead 1985). Note that according to Spinoza, adequacy (the true knowledge of the second kind of knowledge-ratio) equally holds for the part and the whole: "Those things which are common to all, and which are equally in part and in the whole, can only be conceived adequately" (Ethics 2, proposition 38, Spinoza 1985, p. 474). The whole under discussion is the actual absolutely infinite ${ }^{6}$ (Substance) or actual infinities (the Attributes or the Infinite Modes). Spinoza understood, long before Cantor, "what kind of Infinite can be conceived to be greater than another Infinite, without any contradiction" (Letter 12, p. 201).

Still, Huby's approach requires more of our attention. Criticizing Russell for criticizing Kant as to the impossibility of actual infinite and relying upon Cantor's idea of infinite sets, Huby writes that "it can be replied that Cantor's work is a branch of pure mathematics and, as such, can tell us only what may be, not what [actually] is" (Huby 1971, p. 123; italics added). This reply is wrong, for Cantor's theory discovers mathematical pure possibilities that are certainly actualizable under some spatiotemporal and causal circumstances. It is a wrong conclusion that because

6 In mathematics absolute infinite has quite a different meaning, which has to do with all possible ordinals: $\Omega$ is called Absolute Infinity because it is not a relative notion. The line of ordinals leading to $\Omega$ contains all the ordinals, all the possible stages of counting. It is because every possible ordinal occurs before $\Omega$ that $\Omega$ is not really a definite ordinal number" (Rucker 1982, p. 78). 


\section{Philosophy International Journal}

any pure mathematics or mathematical theory is valid for mathematical pure possibilities and their relations, it is not valid for actual reality. This is simply mistaken. There is nothing about pure possibilities as such that prevent them from being actualized whenever actual reality becomes fit to actualize them or when particular spatiotemporal and causal circumstances emerge. There is nothing in the pure sets that precludes them from becoming actual or being applied to actual reality whenever the actual circumstances allow it. On panenmentalist grounds, pure possibilities and their relations are necessary and, to some extent, can be known a priori, whereas actualities, under particular actual circumstances, are contingent. Hence, when the actual circumstances tally with some pure possibilities and the relations between these possibilities as well, there is no reason (rather, cause) why these possibilities would not be actualized. Whenever the actual state of affairs tallies with some mathematical pure possibilities, these possibilities are physically actualized (otherwise such actualities would not be objects for physics, as a science for which mathematical language is inevitable). If a pure set is infinite, there is no reason or cause why it could not be actualized, physically, provided that the actual circumstances allow it. The fact is that astronomical and physical observations have given some support, though not conclusive yet, for the theory of the improved and modified Big Bang cosmology, which in its initial form was already, as Gamow initially realized, compatible with the thoughtexperiment of Hilbert's Hotel, which, in turn, has been an experiment with pure possibilities and their relations. Logically, mathematically, and physically theoretical it is possible that our actual universe is such a weird Hotel. As empirical observations, so far, have partly confirmed that actualization, Huby's critique of the idea of Hilbert's Hotel is groundless, both theoretically and empirically. Furthermore, on panenmentalist grounds, Hilbert's Hotel is really a happy idea.

Huby mentions the distinction between pure mathematics and the application of mathematics to the (actual) world (Huby 1973, p. 187). Note that no physics and no natural science in general can do without applying pure mathematics to physical actualities. This principal truth has been accepted by all physicists since Galileo's great idea about the mathematical language of the Book of Nature. Those mathematical pure possibilities that nature has actualized should not be ignored by us or by Huby. Hilbert's Hotel is a physical-mathematical pure possibility that nature appears to actualize to the extent that astronomical and physical data have confirmed it, though so far only partly. Instead of Huby's distinction between logical possibilities and real possibilities (Huby 1973, p. 186), I prefer to speak of pure possibilities and actual possibilities (actualities) both of which, as mindindependent, are real though in different sense of reality.
Huby also mentions actual entities (1971, p. 127) and, in the same vein, she distinguishes between actual events and potential ones (future potential events); she also distinguishes potential numbers from actual ones (op. cit., p. 129). On panenmentalist grounds, it does not seem "a type-mistake to suppose that there could be as many real objects as there are natural numbers" (ibid), which involves no absurdities. Mentioning Hilbert's Hotel, Huby makes the following reductio: "Hilbert's Hotel, where an infinite number of guests exactly occupy an infinite number of rooms, but where a room can always be found for a new arrival by sending everyone else into the room next door, leaving the first one vacant, becomes a real possibility; similarly, it is possible that all the natural numbers have been written down, or indeed are at this very moment being written down" (Huby 1971, p. 128). Such is not the correct analogy to Hilbert's Hotel. The correct one is to an endless process in which all the natural numbers may be written down. Equally, at any moment, new guests are welcomed in the hotel, and there is no end to them. The pure possibilities that remain open for the Hotel and for its guests are actually not exhaustible.

Because any actuality is a spatiotemporally and causally restricted part of its individual pure possibility, the hard problem of the Platonic middle man in the Parmenides (Huby 1973 , p. 187) is undoubtedly irrelevant to my approach. The aporetic separation (chōrismos) separating the sensuous particular things from the universal Ideas, of which these things are mere "copies" or "instantiations," is not relevant to any panenmentalist individual pure possibility and its actuality as the latter is a limited and conditioned part of the former. In such kind of actualization no regress is involved, let alone a vicious one.

It is not an accident that Hilbert's Hotel is associated with the infinite number of numbers. Numbers and geometrical figures or patterns, which are objects of pure mathematics, are mathematical individual pure possibilities. Contrary to the erroneous idea that numbers and geometrical figures or patterns are abstractions abstracted by various ways from actual reality, panenmentalism considers them quite differently. First, abstractions should be attained according to some models or criteria, which in turn should not be abstracted from actual reality otherwise infinite regress takes place. Second, there are many numbers, such as transfinite numbers, that undoubtedly could not be abstracted from any actual data and they are grasped only thanks to our sheer imagination and mathematical and logical inferences and proofs. The same holds for many geometrical figures or patterns, of which we have no empirical confirmation but they are merely discoveries by our imagination and intellectual abilities. Hence, there is an unbreakable connection between individual pure possibilities and numbers and geometrical entities. 


\section{Gamow's Big Bang Cosmology in Light of Panenmentalism}

Note that Gamow thought that the thought-experiment of Hilbert's Hotel is nicely compatible with Gamow's novel idea of the Big Bang, whereas the adherents of the rival theory, the steady state theory, such as Boyce (whom I have cited above), pointed out the compatibility of this theory with that experiment. Nevertheless, the steady state theory had been proven wrong as a result of the discovery of the cosmic microwave background in 1965 (Kragh 2014, pp. 8-9). This does not affect the weight of the thought-experiment of Hilbert's Hotel, which is nicely compatible with the received improved and modified cosmology of Gamow concerning the Big Bang as well as with the theory of the inflationary expansion of the universe.

Gamow's BigBangmodel in itself is a thought-experiment. The beginning of this model was a mathematical version of it. Gamow used all the available information concerning nuclear reactions but he added to it many reasonable assumptions to simplify the calculations (Hargittai 2011, p. 286). The purely mathematical aspect of such a model is enough to indicate its a priori accessibility (namely, not by means of empirical experience but by means of our imagination and intellect only) and to establish the view that it consists of theoretical pure possibilities and the ways in which they relate to one another (namely, their relationality). As expected, this purely possible model was not readily accepted at the beginning. Even the name "Big Bang" served the opponents of the model-first of all the distinguished astronomer, Frederick Hoyle-to make fun of it. The received view, in contrast, supported at the time the so-called steady state model, whose main proponent was Hoyle. It was Hoyle who coined the name "Big Bang" (Hargittai 2011, p. 287).

One of the empirical, actual anchors of the model was the estimate of the high relative abundance of helium in the universe, which strengthened the credibility of the model's results because the distribution of the light elements was known from entirely independent sources (Hargittai 2011, pp. 286-287). Another, more crucial, empirical or actual anchor to confirm the model was the necessary condition that if the model was correct, there must be "a remnant heat ... which should have stayed around even billions of years after the moment of the Big Bang" (ibid., p. 288). This crucial anchor further confirmed the model, for it was Gamow's prediction, according to the model, that the temperature of the universe is seven kelvins. From two parameters-the age of the universe and the average density of matter in the universe-he estimated this temperature, corresponding to the cosmic microwave radiation (ibid., p. 289). At that stage, the serendipity or the contingency of actual events entered the dramatic scientific scene: in 1964, Arno Penzias and
Robert Wilson, who were not concerned with models about the origin of the universe, reported on "their serendipitous observation of the cosmic microwave radiation amounting to three kelvins," which "was a stunning confirmation of the Big Bang model" (ibid.). As long as the empirical data indicate a temperature that is clearly above zero, these data confirm the model: in the universe there is heat, a remnant of the Big Bang. Hence, three kelvins should be more than enough to serve as a confirmation of the model: "it was definitely established that there is a remnant heat in the universe, giving final and absolutely convincing evidence for the Big Bang model" (ibid., p. 291). It is a mark of a genuine scientist that even if he or she was an ardent opponent of some theory or model, when finally convinced by the evidence, he or she helps to add more evidence to support it. Thus did Frederick Hoyle. When finally convinced about the model, he contributed evidence to acknowledge it and to support its reception.

Nevertheless, empirical findings or actualities do not provide us with an explanation. This is the function of the purely possible model. First, Penzias and Wilson were convinced of the soundness of their measurements (which, unlike calculations, are based on empirical observations), and then they looked for an explanation. They did not connect this explanation with the Big Bang model. At the same time, other scientists studied possible models of the origin of the universe (Hargittai 2011, p. 290; this is another example of the coincidence that has to do with the contingency of actual matters such as events). Penzias and Wilson, the discoverers of cosmic microwave background radiation, found a cosmological interpretation of their empirical observation with the help of some of these scientists.

Istvan Hargittai demonstrates that Gamow brought together seemingly disparate facts, observations, and theories and, from them, he reached a far-reaching conclusion; where he sensed gaps, he augmented the missing links with intuition and imagination (Hargittai 2001, p. 293). In panenmentalist terms, when necessary or even only possible, Gamow ventured to proceed beyond the domain of actualities and empirical data to that of pure possibilities and their relationality. Insatiable curiosity attracted him to quite different areas of interest, whether actual or purely possible, but without an access, owing to his imagination and intuition, to the domain of pure possibilities and their relationality, he could not discover the Big Bang model.

As the Big Bang theory, as such, comprises pure possibilities and the relations between them, it is certainly compatible with the infinite realm of pure possibilities comprising everything actual and purely possible. As for the actual universe, it may continue to endlessly, infinitely expand though it is actually restricted, limited, and conditioned by 


\section{Philosophy International Journal}

spatiotemporal and causal conditions. We know something about the temporal beginning of the universe, which means that we know about it as spatiotemporally and causally restricted and conditioned. If, in the future, telescopes allow us to observe this beginning, we would be sure then about the actual beginning of the universe. In this case, the universe evolves in an infinite space (in the sense of the pure possibility of space) but not in infinite time, as there was a temporal beginning for the universe.

At any given moment, all the rooms in Hilbert's Hotel are occupied, and, at the same time, any vacancy remains open to a new actual state. Thus, the infinite number of the Hotel's rooms comprises both purely possible vacant rooms and actual occupied rooms. At any given moment, the possibilities that are open for the endless, infinite extension of the universe are not exhausted by any actual state of it.

\section{Hilbert's Hotel as Actual, or how it can be Physically Possible}

As for the actual infinite as physically realized, recently, the theory of the inflation of the universe, which Alan Harvey Guth formally proposed in 1980 and which is a modification of the Big Bang theory that Gamow initiated, has gained some support, though this support and the theory itself are still quite controversial ${ }^{7}$. Other attempts to demonstrate how the actual infinity of some kinds of "Hilbert's Hotel" can be physically actualized are the "Quantum Hilbert Hotel" (Potoček et al. 2015) and the "fractal vortex Hilbert's Hotel"

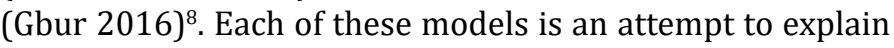
and demonstrate how an infinity, along the lines of Hilbert's Hotel, can be physically actualized. Each of them constructs a unique mechanism for such an actualization. The inflation cosmic models employ mechanisms as Big Bangs (there may be even an infinite number of them!), splitting into multiuniverses (the idea of multiverse, ${ }^{9}$ which is borrowed from

7 Consult: Ijjas, Steinhardt, and Loeb 2017; Steinhardt 2014; Cowen 2014; Ijjas, Steinhardt, and Loeb 2013; and Steinhardt 2011. Steinhardt goes as far as declaring that "it is clear that the inflationary paradigm is fundamentally untestable, and hence scientifically meaningless" (2014, p. 9). Cf. Ade, et al. 2015; Spergel and Keating 2015. Steinhardt criticizes the inflation theory, of which he himself was one of the founders. He especially attacks the idea of multiverse, which he considers as explaining and predicting nothing. According to Steinhardt, even in case that gravitational waves are empirically confirmed, this does not necessarily support the inflation idea.

8 Demonstrating "how the unusual mathematics of transfinite numbers, in particular, a nearly perfect realization of Hilbert's famous hotel paradox, manifests in the propagation of light through fractional vortex plates" (Gbur 2016, p. 222).

9 The idea of multiverse is incompatible with panenmentalism, as it violates the principle of the identity of indiscernibles (in assuming that individua in our world can be or are identical to individua in other worlds), which strictly holds for individual pure possibilities, and as this idea relies upon the notion of possible worlds, which is also incompatible with panenmentalism. Nevertheless, this tells nothing critical of physics; all it quantum mechanics), and others. The quantum Hilbert's Hotel and the fractal vortex Hilbert's Hotel employ quite different mechanisms and each introduces a model of its own, based upon a different physical realm or discipline. Yet, all these different models share the fascinating idea of Hilbert's Hotel.

The yet-controversial inflation theory of the universe is beautifully compatible with the Hilbert's Hotel thoughtexperiment even more than with the standard Big Bang and the expansion theory of the universe, for the inflation theory attempts to show that there can always be "rooms" enough for more and more inflation of the universe. The infinite actual universe thus takes part in a wider infinite realm of individual pure possibilities, each of which is actualizable. The infinite expansion or inflation of the universe indicates that more and more individual pure possibilities, pertaining to the expanded universe, are being actualized each moment. More and more actual new "guests" arrive at the Universe's Hotel which is Hilbert's Hotel. There is no end to the rooms that are always capable of receiving new actual comers.

Let us return to the text by Gamow which reads as follows:

With the new broadening of [the Universe's] horizons a completely new picture emerged: the entire apace of the universe, populated by billions of galaxies, is in a state of rapid expansion, with all its members flying away from one another in high speed. (Gamow 1952, p. 23; italics in the original)

Here we can find a clear suggestion, however initial, for the inflation theory, and the bridging link is, again, the thought-experiment of Hilbert's Hotel. It was Gamow who first compared the expansion of the universe to the expansion of the "surface of a rubber balloon" blown up to a larger and larger size (ibid.). However, to explain this expansion, the inflation cosmology has to discover later the pure possibility of repulsive gravity (Guth 2007). ${ }^{10}$

Whether there was one Big Bang, as the standard Big Bang cosmology stated, or whether there has been an infinite number of them, as versions of the inflation cosmology states, ${ }^{11}$ or a finite number of them, ${ }^{12}$ there are always enough rooms for new "guests" (galaxies, for instance) in the always fully occupied Hilbert's Hotel which is an actual universe, such the one in which, may-be, we live. If there is an infinite number of universes or a finite number of them, each

means is that panenmentalism is incapable of lending any metaphysical support to the idea of multiverse.

10 "The possibility of repulsive gravity arises because, according to Einstein's theory of general relativity, gravitational fields are produced not just by energy or mass densities, but also by pressures" (Guth 2007, p. 81).

11 Guth 2007.

12 Linde and Vanchurin (2010) 
of these universes is an actual Hilbert's Hotel, in which there are always rooms enough for new "guests." On the basis of theories, each of which discovers individual pure possibilities and their relationality, an actual infinity is undoubtedly possible. Nevertheless, are we living in a universe that is a kind of an actual Hilbert's Hotel?

\section{Questioning Actual Infinity}

In spite of the popularity of the notions of cosmic inflation and cosmic actual infinity, there is still place for questioning actual infinity. In spite of this popularity, one should not ignore David Hilbert's denial of the possibility of an actual infinite universe already in 1926:

... we must consider the expanse of the universe to determine whether it embraces anything infinitely large. But here again modern science, in particular astronomy, has reopened the question and is endeavoring to solve it, not by the defective means of metaphysical speculation, but by reasons which are based on experiment and on the application of the laws of nature. Here, too, serious objections against infinity have been found. (Hilbert 1926, p. 186)

Indeed, recently cosmologist Max Tegmark has pronounced serious doubts concerning cosmic actual infinity (Tegmark 2015), for cosmic infinity is unpredictable, immeasurable, and unobservable. ${ }^{13}$ What we observe in the external universe is finite, hence predictable and measurable. Actual infinities are countable but they are not measurable. According to Tegmark, science cannot do without predictability and measure. Hence, we have to get rid of the notions of infinity, at least actual infinity, if we wish to have a realistic picture of the world and make science about it.

Returning to the idea of Hilbert's Hotel, it is quite true that the number of possible new-coming guests and the number of vacant rooms for their accommodation are entirely unpredictable and immeasurable. Yet, actual guests and actually occupied rooms are certainly countable and measurable, however infinite their number. If the cosmos in which we live is such a Hilbert's Hotel, it is unpredictable and immeasurable as long as it is infinitely inflated, whereas the

13 Notwithstanding, when, in fact, relying upon the notion of actual infinite, Tegmark claimed: "The bubbles where inflation has ended are the elements of the Level II multiverse. Each such bubble is infinite in size,... yet there are infinitely many bubbles since the chain reaction never ends. Indeed, if this exponential growth of the number of bubbles has been going on forever, there will be an uncountable infinity of such parallel universes (the same infinity as that assigned to the set of real numbers, say, which is larger than that of the [countably infinite] set of integers). In this case, there is also no beginning of time and no absolute Big Bang: there is, was and always will be an infinite number of inflating bubbles and post-inflationary regions like the one we inhabit, forming a fractal pattern" (Tegmark 2004, p. 466). facts that concerning inflation are observable. In contrast, the model of Quantum Hilbert Hotel refers to prediction and measurements (Potoček et al. 2015, pp. 3-4).

Although predictability plays a crucial role in the success and popularity of both classical and quantum physics, it is, nevertheless, dispensable for science. Chaotic phenomena, for instance, yield deterministic mathematically non-linear equations, and chaos theory is a natural science, even though these equations prevent any prediction in the long run concerning these phenomena. Accepting scientific theory, we habitually rely upon predictability (such is precisely the case of quantum theory), but what we need are explanation and understanding (with which quantum theory appears to be not providing us enough with). Indeed, the cosmic inflationary theory does not provide us with prediction but it provides us with explanation and understanding. It makes sense without making predictions. Such is the case of Hilbert's Hotel. As explaining the possibility of cosmic inflation, it makes sense, it explains and makes this bizarre cosmos understood, though it can never predict about its future inhabitants-the numbers of stars, galaxies, particles, and sub-particles, and yet it retains its scientific other merits, as chaos theory does.

\section{Conclusion}

Only time will tell whether we are living in an actually infinite universe ("actual Hilbert's Hotel"). The current empirical evidence so far is not sufficient to provide us with a well-established or decisive answer. Nevertheless, panenmentalism can explain why and how an infinite actual universe is possible on metaphysical grounds, which in my view are not defective. On these grounds, a purely possible Hilbert's Hotel is, at least, a well-established possibility. Nevertheless, the doubts and questions concerning actual cosmic infinity are still with us. They, too, rest upon considerations that have to do with individual pure possibilities and their relationality as well as with their actualities.

\section{References}

1. Ade PAR (2015) A Joint Analysis of BICEP2/Keck Array and Planck Data, arXiv: 1502.00612v1 [astro-ph.CO].

2. Boyce NW (1972) A Priori Knowledge and Cosmology. Philosophy 47: 67-70.

3. Cowen R (2014) Experts Hail to Gravitational-Wave Revolution, Nature.

4. Ellis GFR, Kirchner U, Stoeger WR (2004) Multiverses and Physical Cosmology, Monthly Notices of the Royal Astronomical Society (MINRAS) 347(3): 921-936. 


\section{Philosophy International Journal}

5. Fine K (2012) Counterfactuals without Possible Worlds. The Journal of Philosophy 109(3): 221-246.

6. Gamow G (1953 [1947]) One, Two, Three ... Infinity: Facts and Speculations of Science. Mentor House, The New American Library, New York.

7. Gamow G (1952) The Creation of the Universe. Viking Press, New York.

8. Gbur G (2016) Fractal Vortex Hilbert's Hotel. Optica 3(3): 222-225.

9. Gilead A (1985) 'The Order and Connection of Things'Are They Constructed Mathematically-Deductively According to Spinoza? Kant-Studien 76: 72-78.

10. Gilead A (1999) Saving Possibilities: An Essay in PhilosophicalPsychology. $80^{\text {th }}$ (Vol), Amsterdam \& Atlanta: Rodopi - Value Inquiry Book Series.

11. Gilead A (2003) Singularity and Other Possibilities: Panenmentalist. 139 (Vol.), Novelties, Amsterdam \& New York: Rodopi - Value Inquiry Book Series.

12. Gilead A (2004) How Many Pure Possibilities Are There? Metaphysica 5(2): 85-104.

13. Gilead A (2005) A Possibilist Metaphysical Reconsideration of the Identity of Indiscernibles and Free Will. Metaphysica 6: 25-51.

14. Gilead A (2008) A Humean Argument For Personal Identity. Metaphysica 9: 1-16.

15. Gilead A (2009) Necessity and Truthful Fictions: Panenmentalist Observations. 202 (Vol.), Amsterdam \& New York: Rodopi - Value Inquiry Book Series.

16. Gilead A (2010) Actualist Fallacies, From Fax Technology to Lunar Journeys. Philosophy and Literature 34(1): 173-187.

17. Gilead A (2011) The Privacy of the Psychological. 233 (Vol.), Amsterdam \& New York: Rodopi - Value Inquiry Book Series.

18. Gilead A (2013a) Shechtman's Three Question Marks: Possibility, Impossibility, and Quasicrystals. Foundations of Chemistry 15: 209-224.

19. Gilead A (2013b) Pure Possibilities and Some Striking Scientific Discoveries. Foundations of Chemistry.

20. Gilead A (2014a) Singularity and Uniqueness: Why Is Our Immune System Subject to Psychological and Cognitive Traits?
21. Gilead A (2014b) Two Kinds of Discovery: An Ontological Account.

22. Gilead A (2014c) Chain Reactions, 'Impossible' Reactions, and Panenmentalist Possibilities. Foundations of Chemistry 16: 201-204.

23. Gilead A (2014d) Two Kinds of Discovery: An Ontological Account.

24. Gilead A (2015a) Self-Referentiality and Two Arguments Refuting Physicalism. International Philosophical Quarterly 55(4): 471-477.

25. Gilead A (2015b) Can Brain Imaging Breach Our Mental Privacy? The Review of Philosophy and Psychology 6(2): 275-291.

26. Gilead A (2015c) Cruelty, Singular Individuality, and Peter the Great. Philosophia 43(2): 337-354.

27. Gilead A (2015d) Neoteny and the Playground of Pure Possibilities. International Journal of Humanities and Social Sciences 5((2): 30-39.

28. Gilead A (2015e) The Twilight of Determinism: At Least in Biophysical Novelties.

29. Gilead A (2016a) Eka-Elements as Chemical Pure Possibilities, Foundations of Chemistry 18(3): 183-194.

30. Gilead A (2016b) The Philosophical Significance of Alan Mackay's Theoretical Discovery of Quasicrystals. Structural Chemistry 28: 249-256.

31. Gilead A (2016c) Personal Singularity and the Significance of Life, Philosophia 44(3): 775-786.

32. Gilead A (2016d) A Philosophical Approach to the Riddle of Michelangelo's Florentine Pietà. Cogent Arts \& Humanities 3: 1-12.

33. Gilead A (2016e) Stanley Milgram's Experiments and the Saving of the Possibility of Disobedience. Journal of Social Sciences 12(2): 88-98.

34. Gilead A (2020) The Panenmentalist Philosophy of Science: From the Recognition of Individual Pure Possibilities to Actual Discoveries. 424 (Vol.), Springer Nature Switzerland AG/Synthese Library.

35. Guth AH (2007) Eternal Inflation and Its Implications.

36. Hargittai I (2011) Drive and Curiosity: What Fuels the Passions for Science. Amherst, N.Y.: Prometheus Books.

37. Hilbert D (1998 [1926]) On the Infinite. In: Benacerraf P, Putnam H (Eds.), Philosophy of Mathematics: Selected 
Readings. Cambridge: Cambridge University Press, pp: 183-201.

38. Hilbert D (2013) Lectures on the Foundations of Arithmetic and Logic 1917-1933. Heidelberg: Springer.

39. Huby PM (1971) Kant or Cantor? That the Universe, If Real, Must Be Finite in Both Space and Time. Philosophy 46: 121-132.

40. Huby PM (1973) Cosmology and Infinity. Philosophy 48: 186-187.

41. Ijjas A, Steinhardt P, Loeb A (2013) Inflationary Paradigm in Trouble After Planck2013. Physics Letters B 723: 261266.

42. Ijjas A. Steinhardt P, Loeb A (2017) Pop Goes the Universe. Scientific American 316: 32-39.

43. Jacobs JD (2010) A Powers Theory of Modality: Or, How I Learned to Stop Worrying and Reject Possible Worlds. Philosophical Studies 151: 227-248.

44. Kragh H (2009) Contemporary History of Cosmology and the Controversy over the Multiverse, Annals of Science 66(4): 529-551,

45. Kragh H (2014) The True (?) Story of Hilbert's Infinite Hotel.

46. Linde A, Vanchurin V (2010) How Many Universes are in the Multiverse?

47. Lowe EJ (2006) Metaphysics as the Science of Essence, presented at the conference The Metaphysics of E. J. Lowe.

48. Menzel C (2016) Actualism. The Stanford Encyclopedia of Philosophy (Winter 2016 Edition).
49. Potoček V (2015) Quantum Hilbert Hotel. Physical Review Letters 115(16): 1-5.

50. Puryear S (2014) Finitism and the Beginning of the Universe. Australasian Journal of Philosophy 92: 619629.

51. Rucker R (1982) Infinity and the Mind: The Science and Philosophy of the Infinite (Boston: Birkhäuser).

52. Spinoza B (1985) The Collected Works of Spinoza. Edwin C (Ed. \& Trans.), Princeton, NJ: Princeton University Press.

53. Spergel D, Keating B (2015) Cosmology: The Oldest Cosmic Light. Nature 518: 170-171.

54. Steinhardt P (2011) The Inflation Debate: Is the Theory at the Heart of Modern Cosmology Deeply Flawed? Scientific American 303: 37-43.

55. Steinhardt P (2014) Big Bang Blunder Bursts the Multiverse Bubble. Nature 510: 9

56. Stoeger WR, Ellis GFR, Kirchner U (2008) Multiverses and Cosmology: Philosophical Issues.

57. Tegmark M (2004) Parallel Universes. In: Barrow JD, Davies PC, Harper CL (Eds.), Science and Ultimate Reality: Quantum Theory, Cosmology and Complexity. Cambridge: Cambridge University Press, pp: 459-491.

58. Tegmark M (2015) Infinity. In: Brockman J (Ed.), This Idea Must Die: Scientific Theories that are Blocking Process. New York: HarperCollins, pp. 48-51.

59. Vetter B (2011) Recent Work: Modality without Possible Worlds. Analysis 71(4): 742-754. 